\title{
Genome wide transcriptome analysis provides bases on colonic mucosal immune system development affected by colostrum feeding strategies in neonatal calves
}

\author{
Zhixiong He $\mathrm{e}^{1,2}$, Amanda Fischer ${ }^{2}$, Yang Song ${ }^{2}$, Michael Steele ${ }^{2^{*}}$ and Le Luo Guan ${ }^{2^{*}}$
}

\begin{abstract}
Background: Delivery of colostrum within the first several hours after birth is vital for establishing successful passive immunity in neonatal dairy calves. However, it is unclear whether a difference in colostrum feeding strategy can affect the development of the calf gastrointestinal tract. The aim of this study was to evaluate the effect of colostrum feeding time within the first $12 \mathrm{~h}$ after birth on the colonic mucosal immune system in neonatal calves using a genome wide transcriptome analysis.

Results: RNA sequencing-based transcriptome analysis of colon tissues collected from 27 male Holstein calves which were randomly assigned to one of three colostrum feeding strategies - (immediately after birth (TRT0); $6 \mathrm{~h}$ after birth (TRT6); $12 \mathrm{~h}$ after birth (TRT12)) - and euthanized at $51 \mathrm{~h}$ of age detected 15,935 $\pm 210,15,332 \pm 415$, and 15,539 \pm 440 expressed genes in the colon under three treatments, respectively. The core transcriptome of the colon included 12,678 genes, with enriched "cellular process" and "metabolic process" as the top two biological functions with 802 of them being immune function related genes. Principal component analysis of the colon transcriptomes did not display a clear separation by colostrum feeding strategy and differential abundance analyses showed no significant difference in the expression of immune related genes among the treatments. Additionally, a weighted gene co-expression network analysis identified 4 significant (|correlation|>0.50 and $p \leq 0.05$ ) gene modules consisting of 122 immune related genes, which were positively or negatively correlated with the abundance of Lactobacillus and Faecalibacterium prausnitzii in the colon.
\end{abstract}

Conclusion: Transcriptome analysis indicates that the development of the colonic mucosal immune system in neonatal calves may be independent of the timing of initial colostrum meal within $12 \mathrm{~h}$ after birth. Our results also provide a molecular understanding of colonic biological function in neonatal calves and extends knowledge on how host gene expression profiles are associated with the abundance of specific bacterial groups in the colon.

Keywords: Calf, Colon, Colostrum feeding, RNA-sequencing

\section{Background}

Due to the presence of syndesmochorial placenta in dairy cows, immunoglobulins (Igs) cannot be transferred from the dam to the fetus, which results in the newborn calf being agammaglobulinemic [1]. Ingestion and absorption of adequate amounts of colostral IgG, known as

\footnotetext{
* Correspondence: masteele@ualberta.ca; Iguan@ualberta.ca

${ }^{2}$ Department of Agriculture, Food and Nutritional Sciences, University of

Alberta, Edmonton T6G 2P5, Canada

Full list of author information is available at the end of the article
}

the passive transfer of immunity, is a prerequisite for neonatal calf health and survival [2-4]. For instance, inadequate IgG absorption from colostrum is associated with higher risks of enteric infections caused by rotavirus and Cryptosporidium spp. [5]. It has been suggested that the timely delivery of high quality and quantity colostrum within the first $24 \mathrm{~h}$ after birth is vital for the establishment of passive immunity in calves $[6,7]$. Recent studies have shown that substantially larger IgG intakes are required when feeding a colostrum

(c) The Author(s). 2018 Open Access This article is distributed under the terms of the Creative Commons Attribution 4.0 International License (http://creativecommons.org/licenses/by/4.0/), which permits unrestricted use, distribution, and reproduction in any medium, provided you give appropriate credit to the original author(s) and the source, provide a link to the Creative Commons license, and indicate if changes were made. The Creative Commons Public Domain Dedication waiver (http://creativecommons.org/publicdomain/zero/1.0/) applies to the data made available in this article, unless otherwise stated. 
meal more than $2 \mathrm{~h}$ after birth in order to achieve successful passive transfer [8], suggesting the importance of feeding colostrum to newborn calves immediately after birth. In addition to containing IgG, colostrum is also rich in a variety of bioactive components, such as growth factors, hormones and prebiotics [9], which are important for cellular growth and differentiation, and influence gut function, metabolism and immune reactions $[10,11]$. Under practical management, not all calves can be fed within a few hours after birth. Therefore, we hypothesized that delaying colostrum feeding, even within the first hours after birth, would result in a delayed effect on the development of the mucosal immune system in the gastrointestinal tract.

In pre-ruminants, the dietary substrates that are not digested in the stomach and small intestine reach the colon and bacterial fermentation of nutrient substrates can produce short chain fatty acids (SCFAs). It has been reported that the SCFAs including butyrate and propionate play important roles in regulation gut physiology, barrier function, immune system and inflammatory response [12-14], suggesting that the colon functions as a site of immune response and the microbes in the colon could directly or indirectly affect the immune function of the colon via microbial production of SCFAs. However, knowledge on the functional development of the colon in pre-ruminants is limited and the functional development of the colon in neonatal calves has not been investigated. The objective of this study was to characterize the function of colon tissues during early life using RNA-sequencing (RNA-seq) based transcriptome analysis and to evaluate whether colonic mucosal immune system development of neonatal calves can be affected by a delayed colostrum feeding.

\section{Methods}

\section{Animal, feeding and sample collection}

The Livestock Care Committee of the University of Alberta reviewed and approved all procedures for the animal trial (AUP00001595), which complied with the guidelines of the Canadian Council of Animal Care [15]. Calves were born at the Dairy Research and Technology Centre of the University of Alberta from February to September of 2016, and only the male calves from a singleton birth with a body weight between 35 and $55 \mathrm{~kg}$ were chosen for the experiment. As a result, 27 male Holstein calves, 12 from primiparous heifers $(\mathrm{H})$ and 15 from multiparous cows $(C)$ with an average birth weight of $42.6 \pm 4.21 \mathrm{~kg}$ (mean $\pm \mathrm{SD})$, were used in the current study. Immediately after birth, the calves were removed from the dam (without physical contact), ear tagged, weighed and housed in individual pens with straw bedding. The calves were randomly assigned to one of 3 feeding strategies for the initial colostrum meal after birth $(7.5 \%$ of birth body weight; $n=9$ each group, 4 from $\mathrm{H}$ and 5 from $\mathrm{C}$ ): 1) fed immediately after birth (TRT0); 2) fed at $6 \mathrm{~h}$ after birth (TRT6); or 3) fed at $12 \mathrm{~h}$ after birth (TRT12). Pooled and pasteurized colostrum (62 g/L of IgG; Saskatoon Colostrum Company Ltd.; Saskatoon, SK, Canada) was thawed from frozen in a water bath and heated to $39{ }^{\circ} \mathrm{C}$ prior to feeding. At their respective feeding time, colostrum was tubed to calves using an esophageal tube feeder. Twelve hours after the colostrum meal, all calves were fed a milk replacer diet $(260 \mathrm{~g} / \mathrm{kg}$ crude protein, $180 \mathrm{~g} / \mathrm{kg}$ crude fat; Excel Pro-Gro Calf Milk Replacer, Grober Nutrition, Cambridge, Ontario, Canada) at a volume of $2.5 \%$ of birth body weight every $6 \mathrm{~h}$ of life. There was no difference in calf birth weight among colostrum treatments and the sample collection was performed following the procedures described in the study of Fischer et al. [16]. Briefly, the calves were euthanized with an intravenous injection of pentobarbital sodium at $51 \mathrm{~h}$ of age, and 5 -cm segments of the colon were collected distal to the ileo-cecal junction during dissection. The samples, rinsed three times with PBS buffer to remove ingesta, were immediately frozen in liquid- $\mathrm{N}_{2}$ and stored at $80{ }^{\circ} \mathrm{C}$ freezer.

\section{RNA extraction and sequencing}

Total RNA was extracted from frozen colon tissues using a mirVana total RNA Isolation Kit (Ambion, Carlsbad, CA, USA) following the manufacturer's instruction. Firstly, ground frozen tissue $(\sim 100 \mathrm{mg})$ was mixed thoroughly with 10 volumes of mirVana Lysis/Binding Solution by vortexing for $30 \mathrm{~s}$. Samples were then homogenized by a Precellys 24 homogenizer (Bertin Technologies, Montigny, France) for $30 \mathrm{~s}$ twice. After homogenization, an equal volume of Acid-Phenol:Chloroform (Ambion) was added, followed by vortexing the mixture for $1 \mathrm{~min}$ and centrifugation for $5 \mathrm{~min}$ at $10,000 \times \mathrm{g}$. The resulting aqueous solution was mixed thoroughly with 1.25 volumes of $100 \%$ ethanol and filtered through a mirVana column in a $700 \mu \mathrm{l}$ aliquot twice. After the column was washed 3 times using the given wash solution, the RNA remaining in the column were eluted in nuclease-free water at $95{ }^{\circ} \mathrm{C}$. The quality and quantity of RNA were measured by an Agilent 2200 TapeStation (Agilent Technologies, Santa Clara, CA, USA) and a Qubit 2.0 Fluorometer (Invitrogen, Carlsbad, CA, USA), respectively. All RNA samples had least a 1.8 ratio of A260nm/A280nm measured by Nanodrop (Nanodrop Technologies, Wilmington, DE, USA) and had an integrity number of 7.0 or higher. All RNA samples were then stored at $-80{ }^{\circ} \mathrm{C}$.

Total RNA (100 ng) from each sample was used for library construction using TruSeq RNA Library Prep Kit v2 (Illumina, San Diego, CA, USA) according to the manufacturer's instructions. Firstly, total RNA was fragmented 
and the first and second strand cDNA were synthesized through reverse transcription. The cDNA was then subjected to end repair and 3 '-end adenylation, followed by ligation of index adapters and PCR enrichment $\left(98^{\circ} \mathrm{C}\right.$ for $30 \mathrm{~s}$, followed by 15 cycles of: $98{ }^{\circ} \mathrm{C}$ for $10 \mathrm{~s}, 60{ }^{\circ} \mathrm{C}$ for $30 \mathrm{~s}, 72{ }^{\circ} \mathrm{C}$ for $30 \mathrm{~s}$, and $72{ }^{\circ} \mathrm{C}$ for $5 \mathrm{~min}$; the final products were held at $\left.4{ }^{\circ} \mathrm{C}\right)$. Libraries (250-270 bp) were verified using an Agilent 2200 TapeStation (Agilent Technologies) and quantified with a Qubit fluorometer using a Qubit dsDNA HS Assay Kit (Invitrogen). The individual indexed libraries were then pooled and sequenced at Génome Québec (Montréal, Canada) on the Illumina HiSeq 4000 platform (Illumina) to obtain 100 bp paired-end reads.

\section{RNA sequencing data processing and normalization}

Sequencing reads that did not pass the Illumina chastity filter in CASAVA 1.8 (Illumina) were discarded and filtered reads were then aligned to the reference bovine genome (UMD 3.1) using Tophat 2.1.1 [17, 18]. The BAM alignment files generated from Tophat were sorted using Samtools (v1.4) [19] and converted to SAM files. The SAM files were analyzed using HTSeq-count to obtain gene read counts based on the annotation from ENSEMBL bovine genes [20]. Reads that were assigned to more than one gene were not counted by HTSeq-count. To compare the expression profiles, the read counts of each gene were normalized to counts per million (CPM) using the following formula: $\mathrm{CPM}=$ (gene read counts/total mapped counts per library) $\times 1,000,000$. Principal Component Analysis (PCA) was conducted with cluster samples based on gene expression data using $\mathrm{R}$ ( $\mathrm{R}$ version 3.4.2).

\section{Transcriptome profiles and differential expression analysis}

Genes with at least one CPM in all calves were considered as core genes. The functions of the core genes in the colon were annotated using protein annotation through evolutionary relationship (PANTHER; Version 12.0, released July 10, 2017) gene list analysis tool [21]. Functional classification of genes in categories such as molecular function, biological process and cellular component, were based on gene ontology (GO) annotations.

Differentially expressed (DE) genes were analyzed using the bioinformatics package edgeR (v3.5) [22]. The sequencing data was firstly filtered by keeping genes with at least one CPM in $50 \%$ of the samples per treatment group (5 out of 9 animals in each group). To investigate the overall colostrum treatment and parity effect on colonic transcriptome profiles, 4 contrasts including TRT6 vs. TRT0, TRT12 vs. TRT0, TRT12 vs. TRT6, and $\mathrm{C}$ vs. $\mathrm{H}$ were conducted. Another 9 contrasts including TRT6_H vs. TRT0_H, TRT12_H vs. TRT0_H, TRT12_H vs. TRT6_H, TRT6_C vs. TRT0_C, TRT12_C vs. TRT0_C, TRT12_C vs. TRT6_C, TRT0_C vs.
TRT0_H, TRT6_C vs. TRT6_H, and TRT12_C vs. TRT12_H were also conducted to identify transcriptomic differences within colostrum treatment and parity. $p$ values were multiple-test corrected for false discovery rate (FDR) using the Benjamini and Hochberg method, and significant $\mathrm{DE}$ genes were declared at fold change $\geq 1.2$ and $\mathrm{FDR} \leq 0.10$.

\section{Analysis of specific genes related to mucosal immune system}

To investigate the colonic mucosal immune system development affected by colostrum treatment and parity, the sequencing data of immune related genes was further analyzed. The immune related gene list was obtained from ImmPort database [23] based on their categorized function in cytokine, cytokine receptor, interleukin (IL), interleukin receptor, interferon, interferon receptor, tumor necrosis factor (TNF) family member, TNF family member receptor, transforming growth factor (TGF-b) family member, TGF-b family member receptor, chemokine, chemokine receptor, $\mathrm{T}$ cell receptor (TCR) signaling pathway, breakpoint cluster region (BCR) signaling pathway, natural killer cell, antigen processing and presentation, or antimicrobial (shown in Additional file 1). In addition, IL10, signal transducers and activators of transcription 3 (STAT3), nuclear factor kappa B 1 (NFKB1), tumor necrosis factor (TNF), tolllike receptors $(T L R) 2, T L R 4$, claudin 4 (CLDN4) and peptidoglycan recognition protein 1 (PGLYRP1) genes, which have been previously investigated in dairy calves, were also included as the representative genes of intestinal mucosal immunity [24-27].

\section{Gene co-expression network analysis}

To identify the modules and key genes associated with bacterial populations in the colon, a gene co-expression network analysis was conducted based on their relationship with the copy numbers of Lactobacillus, Faecalibacterium prausnitzii (F. prausnitzii) and Bifidobacterium associated with the colon tissue and content using Weighted Gene Co-expression Network Analysis (WGCNA; v1.49) [28]. The microbial data were obtained from Fischer et al. [16] who used the same colon samples and reported above bacterial abundances using quantitative PCR. Automatic network construction and module detection function (blockwiseModules within the WGCNA) were used to generate subsets of genes, and the modules were correlated with abundance of Lactobacillus, F. prausnitzii and Bifidobacterium in the colon based on the following major parameters: minModuleSize of 30, reassign Threshold of 0 , and mergeCutHeight of 0.30 . The resulting gene modules were assigned with different color names by the software and Module-Trait relationships were calculated by Pearson correlation. Modules with significant 
correlations (|correlation $\mid>0.50$ and $P \leq 0.05)$ were kept and further investigated.

\section{Results}

Colonic transcriptome profiles of neonatal dairy calves

A total of 877 million paired-end reads were obtained from all 27 samples, with an average of $32.5 \pm 9.1$ million reads per sample (Additional file 2). The overall mapped rate to the bovine reference genome (UMD 3.1) was $87.4 \pm 6.2 \%$. The total number of genes prior to any data filtering across the 27 samples was 24,617 with 12,678 core genes (genes with at least one CPM in all the samples) identified. From these core genes, 11,613 genes could be annotated by PANTHER and functional analysis of the core genes showed that the top biochemical processes (Fig. 1a) were "cellular process" (29.4\% of genes) and "metabolic process" (26.3\% of genes); the top molecular functions (Fig. 1b) were "catalytic activity" (39.8\% of genes) and "binding" (39.0\% of genes); the top cellular components (Fig. 1c) were "cell part" $(40.0 \%$ of genes) and "organelle" (25.8\% of genes). The symbol and
CPM of genes used for core functional analysis are listed in Additional file 3.

\section{Colonic transcriptome changes in response to delayed colostrum feeding in neonatal dairy calves}

The number of expressed genes in the colon of TRT0, TRT 6 and TRT12 calves was 15,935 $\pm 210,15,332 \pm 415$, and $15,539 \pm 440$, respectively. The transcriptome profiles did not reveal a clustering pattern either by colostrum treatment or parity (Fig. 2a). Principal component analysis of the transcriptome profiles also did not display a clear separation either by colostrum treatment or parity (Fig. 2b). Differential gene expression analyses across/ within colostrum treatment and parity showed that almost none of the genes passed a FDR correction for multiple testing, with the exception that regulator of $G$ protein signaling 2 (RGS2) was up-regulated (fold change $=8.04$, FDR $=0.032)$ in the colon of TRT6_H calves when compared to that of TRT0_H, down-regulated (fold change = $6.74, \mathrm{FDR}=0.002$ ) in the colon of TRT12_C calves when compared to that of TRTO_C, and up-regulated (fold

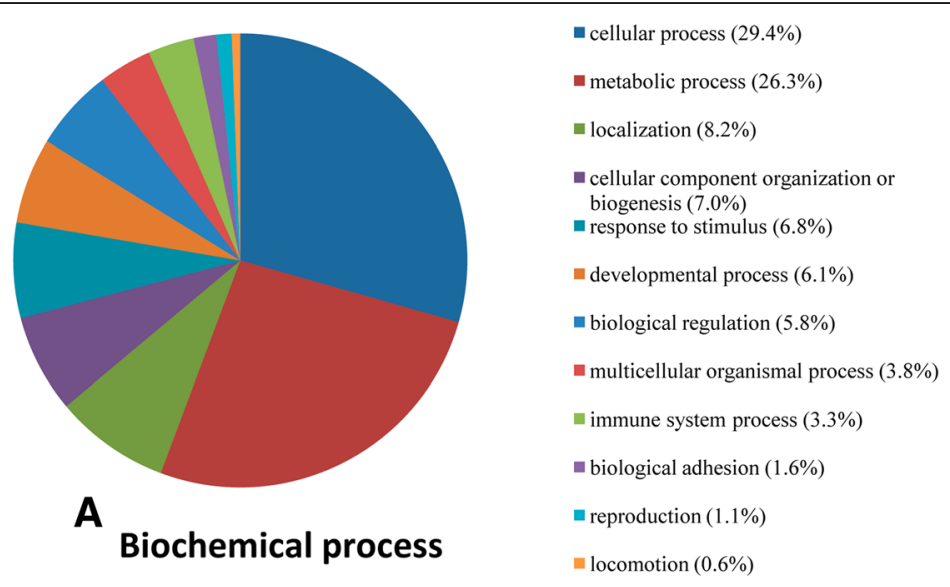

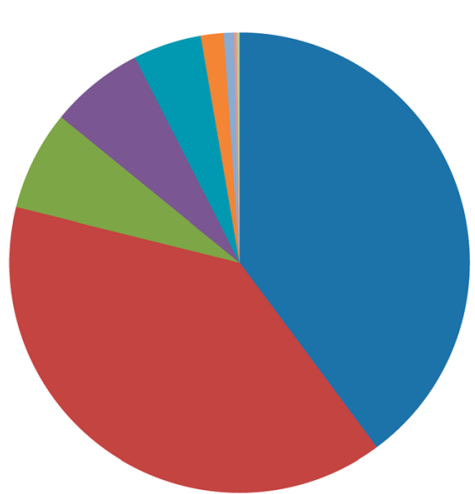

B

Molecular function
- catalytic activity (39.8\%)

- binding $(39.0 \%)$

structural molecule activity $(7.0 \%)$

- transporter activity (6.6\%)

- receptor activity $(4.8 \%)$

mignal transducer activity (1.6\%)

Iranslation regulator activity $(0.7 \%)$

w channel regulator activity $(0.2 \%)$

antioxidant activity $(0.2 \%)$

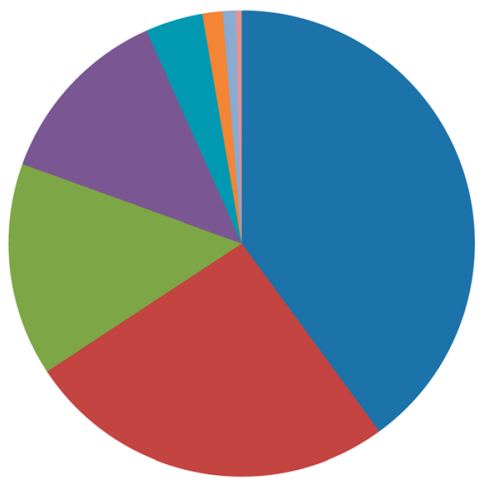

m cell part $(40.0 \%)$

- organelle (25.8\%)

macromolecular complex $(14.8 \%)$

membrane (12.8\%)

In extracellular region (4.0\%)

" extracellular matrix (1.4\%)

In cell junction $(0.9 \%)$

Innapse $(0.4 \%)$

Fig. 1 Functional classification of the core genes in the colon of dairy calves as identified by PANTHER. The percentage of core genes involved in (a) Biological processes, (b) Molecular functions, and (c) cellular components 
A
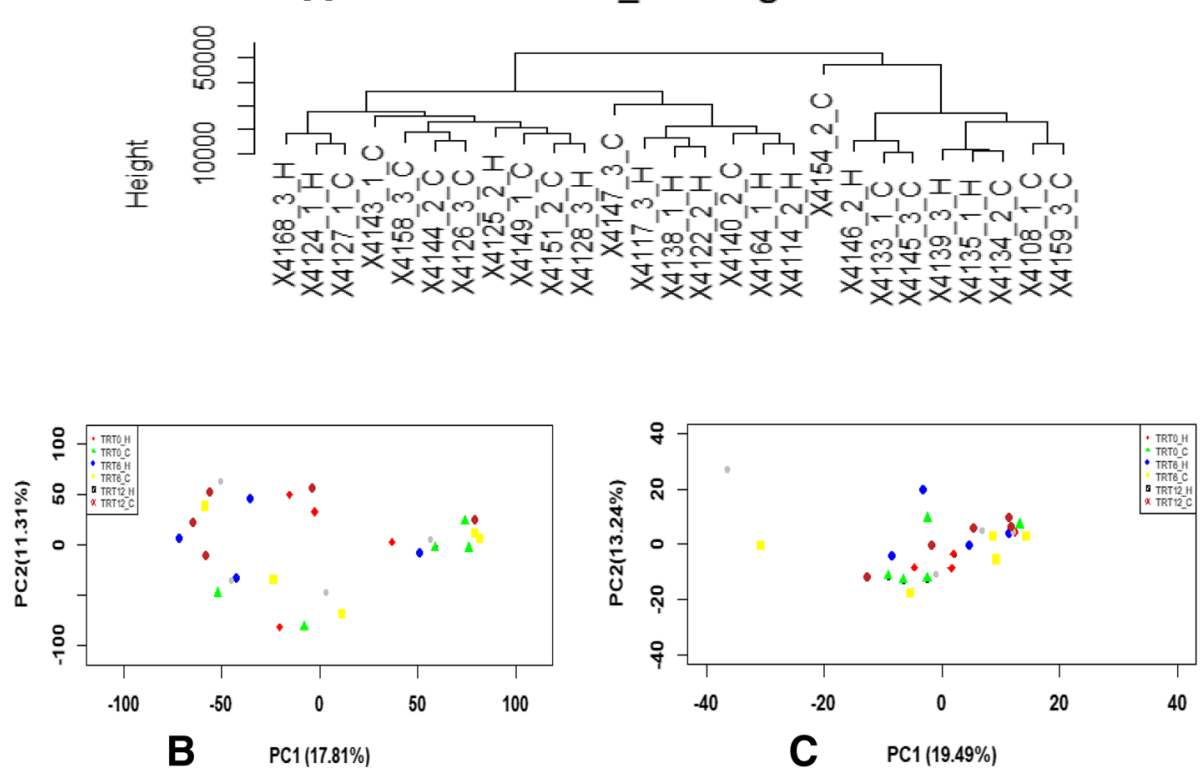

Fig. 2 Cluster and PCA plot of transcriptome profiles. a Hierarchical cluster of transcriptomes. ID represents one sample from animal. For instance, " $\times 4168 \_3 \_H$ " means the sample from animal No. $\times 4168$ which was assigned to the third group (TRT12) and from primiparous heifers (H). $\mathbf{b}$ PCA plot of transcriptomes for the colon samples from TRT0_H (red color), TRT0_C (green color), TRT6_H (blue color), TRT6_C (yellow color), TRT12_H (grey color) and TRT12_C (brown color). The $\mathrm{X}$ and $\mathrm{Y}$-axis represent the first two principle components. The percentage value in the bracket represents the percentage of variance explained by that principle component. c PCA plot of the immune-related genes

change $=8.25$, FDR $=0.006)$ in the colon of TRT0_C calves when compared to that of TRT0_H. In addition, the expression of Chemokine ( $\mathrm{C}-\mathrm{C}$ motif) ligand 14 (CCL14) was up-regulated (fold change $=4.26$, FDR $=$ 0.029) in the colon of TRT6_C when compared to that in TRT6_H (Table 1, Fig. 3).

\section{Assessment of the expression of genes involved in immune function}

A total of 802 genes were identified to be related to immune function and were subjected to further analysis (Additional file 1). Similar to the whole transcriptome, the expression of these immune related genes did not display a clear separation either by colostrum treatment or parity (Fig. 2c). The top 10 expressed immune related genes, based on an averaged CPM value, were $A H N A K$ nucleoprotein (AHNAK), LDL receptor related protein (LRP1), desmin (DES), actin gamma 1 (ACTG1), heat shock protein family A member 8 (HSPA8), solute carrier family 40 member 1 (SLC4OA1), alpha-2-macroglobulin (A2M), calnexin (CANX), calreticulin (CALR) and tropomyosin 2 (TPM2), and the top four expressed immune related genes were all categorized as antimicrobials (Table 2). The expression profiles of these genes are plotted in Fig. 4 and their mean, SD, min, max values of CPM are presented in Table 2.
Table 1 Differentially expressed genes affected by colostrum treatment and dam parity

\begin{tabular}{llll}
\hline & DE gene & Log (FC) & FDR \\
\hline Within parity & & & \\
TRT6_H vs. TRT0_H & RGS2 & 3.01 & 0.032 \\
TRT12_H vs. TRT0_H & NS & NS & NS \\
TRT12_H vs. TRT6_H & NS & NS & NS \\
TRT6_C vs. TRT0_C & NS & NS & NS \\
TRT12_C vs. TRT0_C & RGS2 & -2.75 & 0.002 \\
TRT12_C vs. TRT6_C & NS & NS & NS \\
Within treatment & & & \\
TRT0_C vs. TRT0_H & RGS2 & 3.04 & 0.006 \\
TRT6_C vs. TRT6_H & CCL14 & 2.09 & 0.029 \\
TRT12_C vs. TRT12_H & NS & NS & NS \\
Across parity & & & NS \\
TRT6 vs. TRT0 & NS & NS & NS \\
TRT12 vs. TRT0 & NS & NS & NS \\
TRT12 vs. TRT6 & NS & & NS \\
Across treatment & & NS \\
C vs. H & Note: H = primiparous heifers; C= multiparous cows; RGS2=regulator of G-protein
\end{tabular}

Note: $\mathrm{H}=$ primiparous heifers; $\mathrm{C}=$ multiparous cows; $R G S 2$ = regulator of $\mathrm{G}$-protein signaling 2; NS = not significant; CCL14 = chemokine (C-C motif) ligand $14 ; \mathrm{FC}=$ fold change; cutoff for $\mathrm{DE}$ genes was $\mathrm{FDR}<0.10$, and $|\mathrm{FC}|>1.2$ 
A

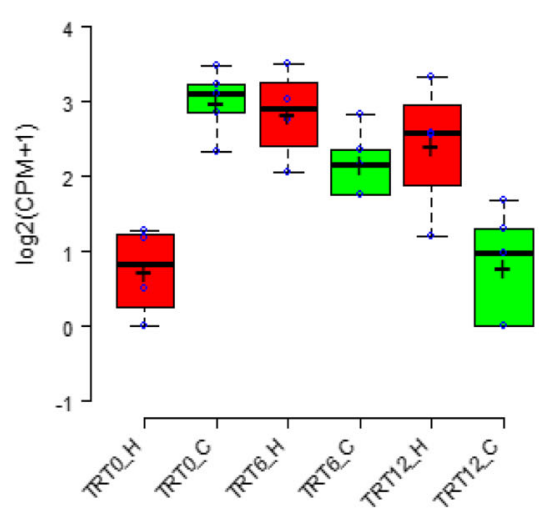

B

CCL14

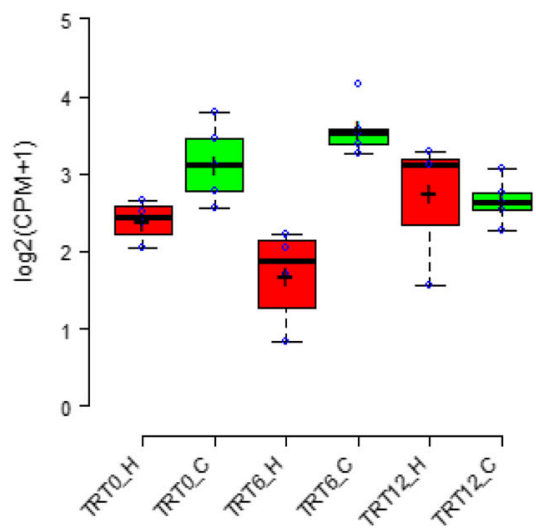

Fig. 3 The expression level of RGS2 (a) and CCL14 (b) in dairy calves with varying colostrum feeding time

Exploration of modules and key genes associated with bacterial colonization in the colon of neonatal dairy calves

To investigate if there was a relationship between the expression of genes and the bacterial populations in the colon, a network-based analysis WGCNA was further conducted. A total of 15,578 genes remained for the input of WGCNA. After setting a minimum module size of 30 and merging modules with highly correlated eigengenes, a total of 23 modules were found. All modules were assigned a color and depicted in the hierarchical clustering dendrogram outlined in Additional file 4. Among the 23 co-expressed gene modules, 1 and 3 modules were significantly (|correlation $\mid>0.50$ and $P \leq 0.05$ ) correlated with the abundance of Lactobacillus and F. prausnitzii in the colon, respectively (Table 3, Additional file 5). For Lactobacillus, the Greenyellow module (264 genes including 11 immune related genes) had a negative correlation $(r=-0.52$ and $p=0.006)$ with its abundance in the colon tissue. For $F$. prausnitzii, the Red module (863 genes including $46 \mathrm{im}$ mune related genes) had a positive correlation ( $r=0.60$ and $p=0.001$ ) with its abundance in the colon content, and the Lightgreen module (62 genes including 2 immune related genes) and the Grey module (1505 genes including $63 \mathrm{im}$ mune related genes) had a negative correlation $(r=-0.82$ and $p<0.001$ for the Lightgreen module; $r=-0.68$ and $p<$ 0.001 for the Grey module) with its abundance in the tissue. There was no significant correlation detected between the modules and Bifidobacterium abundance in the colon. The gene list and name within each module significantly declared are available in Additional file 6.

\section{Discussion}

To our knowledge, this is the first study reporting the transcriptome profiles of the colon and their changes in

Table 2 Expression summary statistics (difference between individual real CPM and the average CPM) of the representative immune related genes

\begin{tabular}{|c|c|c|c|c|c|c|c|}
\hline & Mean & SD & Min & Max & Range & Rank & Category \\
\hline AHNAK & 3252 & 327 & 2655 & 3927 & 1271 & 1 & Antimicrobials \\
\hline$L R P 1$ & 1434 & 234 & 995 & 1803 & 808 & 2 & Antimicrobials \\
\hline DES & 1433 & 394 & 923 & 2285 & 1362 & 3 & Antimicrobials \\
\hline ACTG1 & 1385 & 217 & 935 & 1843 & 908 & 4 & Antimicrobials \\
\hline $1 L 10$ & 0.8 & 0.8 & 0.0 & 3.0 & 3.0 & 780 & Antimicrobials, Cytokines, TCR signaling Pathway \\
\hline STAT3 & 268 & 51 & 196 & 385 & 189 & 26 & Antimicrobials \\
\hline NFKB1 & 57 & 12 & 35 & 97 & 62 & 153 & Antimicrobials, BCR Signaling Pathway, TCR signaling Pathway \\
\hline TNF & 2.1 & 1.7 & 0.0 & 8.1 & 8.1 & 686 & Antimicrobials, Cytokines, Natural Killer Cell \\
\hline$T L R 2$ & 7.2 & 2.5 & 3.9 & 13.7 & 9.8 & 525 & Antimicrobials \\
\hline TLR4 & 17.5 & 6.5 & 7.8 & 40.3 & 32.5 & 389 & Antimicrobials \\
\hline CLDN4 & 44 & 19 & 10 & 92 & 82.3 & 210 & Antimicrobials \\
\hline PGLYRP1 & 4.7 & 4.2 & 0.0 & 13.2 & 13.2 & 586 & Antimicrobials \\
\hline
\end{tabular}




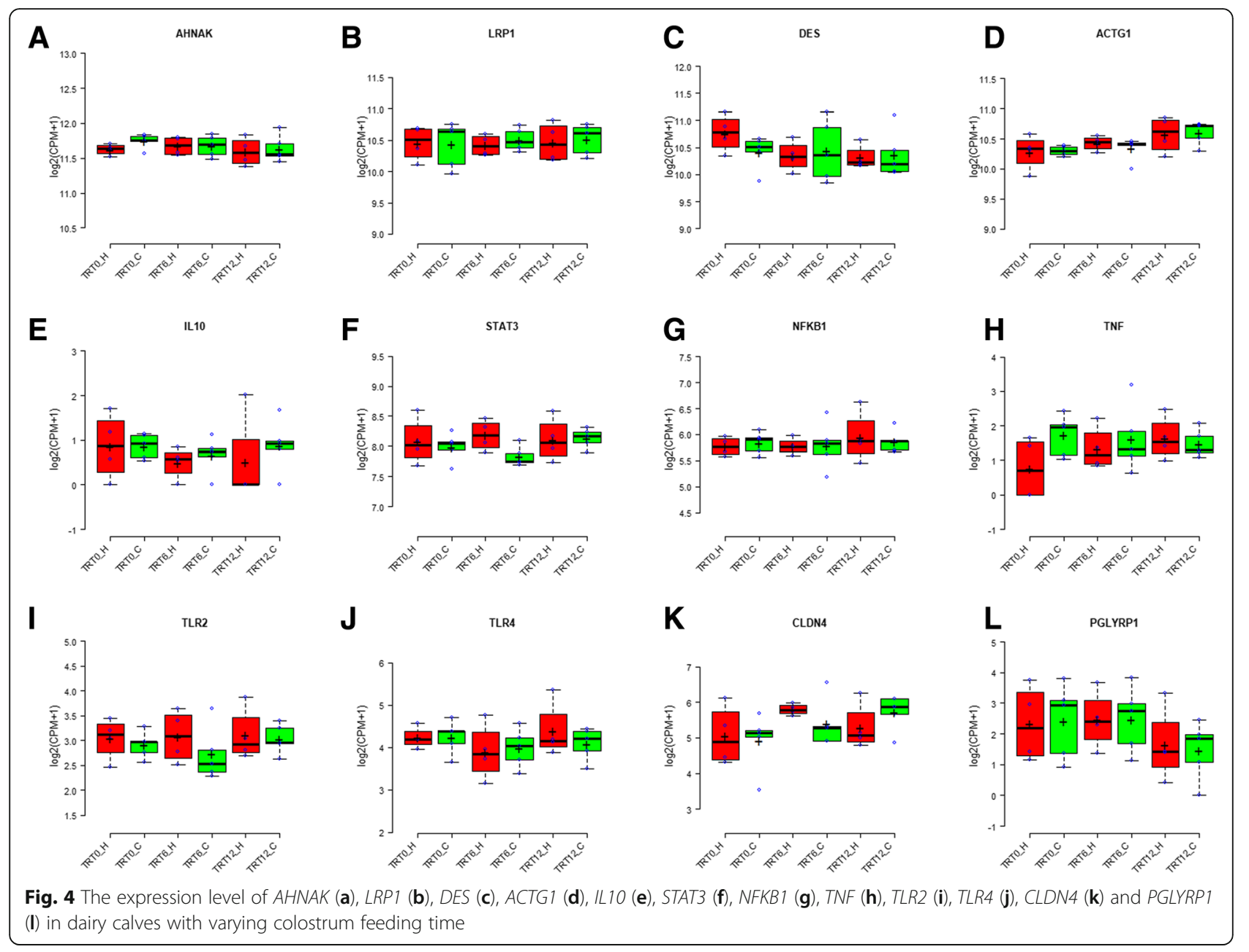

response to colostrum feeding strategies in neonatal calves. In ruminants, the function of the colon has been overlooked in the past; however, a recent study revealed that fermentation in the colon could provide energy to the host through microbial production of SCFAs in pre-weaned calves [14], suggesting that the colon plays an important role in nutrient metabolism. In the present study, transcriptome analysis revealed that the top biochemical processes of the colon in neonatal calves are involved in cellular and metabolic processes, suggesting that the colon plays an important role in nutrient metabolism in neonatal calves - a finding in line with other animal models which highlight the role of the production of SCFAs by the colonic microbiota to maintain energy homeostasis [29]. Interestingly, gene expression during the maturation of the colon in rats has been shown to be up- or down-regulated by an antibiotic treatment from day 7 of life until day 17 or 21, and their functions were related to ion transport processes [30] an outcome which was not observed in the neonatal calves in the present study. This may suggest the presence of age-related functional development and maturation of the colon in neonates, which was not observed in the present study due to the colonic transcriptome profiles being analyzed only at $51 \mathrm{~h}$ of life, and not any further.

The gastrointestinal tract acts as the first defense against pathogenic infections by providing physical barriers and activating innate and adaptive immune responses [31], which is particularly important in agammaglobulinemic calves in order to prevent infection and sickness, such as diarrhea. Song et al. [14] suggested that the colon of dairy calves harvest an abundance of diverse microbes soon after birth, which might be directly or indirectly associated with the immune function of colon. Thus, it was not surprising that immune function $(802$ genes) was identified with the core transcriptome of the colon, accounting for $7.03 \%$ of the total core gene expressions. Immune function in other compartments of the intestine of neonatal calves (at birth, 7, 21 and 42 days of life), such as the ileum and jejunum, has also been identified by Liang et al. [32] via RNA-seq based transcriptome analysis. However, of the 2499 immune related genes obtained from ImmPort (gene list shown 
Table 3 Gene modules correlated with the bacterial abundance in the colon identified using a weighted gene co-expression network analysis

\begin{tabular}{|c|c|c|c|c|c|c|c|}
\hline Module & Gene number (number of immune genes) & lac_C_P & lac_T_P & fea_C_P & fea_T_P & bif_C_P & bif_T_P \\
\hline Black & 488 & NS & NS & NS & NS & NS & NS \\
\hline Blue & 2332 & NS & NS & NS & NS & NS & NS \\
\hline Brown & 2102 & NS & NS & NS & NS & NS & NS \\
\hline Cyan & 140 & NS & NS & NS & NS & NS & NS \\
\hline Darkgreen & 60 & NS & NS & NS & NS & NS & NS \\
\hline Darkred & 60 & NS & NS & NS & NS & NS & NS \\
\hline Green & 955 & NS & NS & NS & NS & NS & NS \\
\hline Greenyellow & $264(11)$ & NS & $\begin{array}{l}-0.52 \\
(p=0.006)\end{array}$ & NS & NS & NS & NS \\
\hline Grey & 1505 (63) & NS & NS & NS & $\begin{array}{l}-0.68 \\
(p=9.00 \mathrm{E}-05)\end{array}$ & NS & NS \\
\hline Grey60 & 79 & NS & NS & NS & NS & NS & NS \\
\hline Lightcyan & 88 & NS & NS & NS & NS & NS & NS \\
\hline Lightgreen & $62(2)$ & NS & NS & $\begin{array}{l}-0.82 \\
(p=1.00-07)\end{array}$ & NS & NS & NS \\
\hline Lightyellow & 62 & NS & NS & NS & NS & NS & NS \\
\hline Magenta & 367 & NS & NS & NS & NS & NS & NS \\
\hline Midnightblue & 93 & NS & NS & NS & NS & NS & NS \\
\hline Pink & 435 & NS & NS & NS & NS & NS & NS \\
\hline Purple & 351 & NS & NS & NS & NS & NS & NS \\
\hline Red & $863(46)$ & NS & NS & $\begin{array}{l}0.60 \\
(p=0.001)\end{array}$ & NS & NS & NS \\
\hline Royalblue & 62 & NS & NS & NS & NS & NS & NS \\
\hline Salmon & 189 & NS & NS & NS & NS & NS & NS \\
\hline Tan & 204 & NS & NS & NS & NS & NS & NS \\
\hline Turquoise & 3730 & NS & NS & NS & NS & NS & NS \\
\hline Yellow & 1086 & NS & NS & NS & NS & NS & NS \\
\hline
\end{tabular}

Note: Only the number of immune genes in significantly correlated modules were shown in the brackets; NS = not significant; lac_C_p = Lactobacillus population from the colon content; lac_T_ $p=$ Lactobacillus population from the attached colon; fea_C_ $p=$ Faecali bacterium prausnitzii population from the colon content; fea_T_$p=$ Faecali bacterium prausnitzii population from the attached colon; bif_C_ $p=$ Bifidobacterium population from the colon content; bif $\_$T_ $p=$ Bifidobacterium population from the attached colon

in Additional file 1), our study only found 802 genes expressed in the colon, which may reflect tissue-specific gene expression patterns and demonstrate that the immune system of the colon was still immature (with $67.9 \%$ of the immune related genes being not expressed) at 2 days after birth. Such finding may provide molecular evidence that newborn calves are susceptible to gastrointestinal pathogenic infections [33]. In fact, it has been proposed that the susceptibility of newborns to pathogens is not from any inherent inability to mount an immune response, but rather, is caused by the fact that their immune system is unprimed [34]. The identification of the genes expressed in the mucosal immune system may also provide a molecular basis to suggest that the neonatal intestinal mucosal immune system is undergoing rapid changes during the early postnatal developmental period $[32,35]$.
In this study, very few differences were detected in the expression levels of the overall transcriptome and immune related genes of the colon based on varying colostrum feeding strategies. Colostrum contains a variety of bioactive components such as growth factors and hormones [9]. The lack of localized beneficial effects in TRT0 calves suggested that colostrum feeding time within $12 \mathrm{~h}$ after birth may result in no difference for the colonic development since the colon of calves was primed the same amount of bioactive components within $51 \mathrm{~h}$ of lifetime prior to the euthanasia. Moreover, passive transfer of IgG is generally considered successful when serum IgG concentrations in dairy calves are $10 \mathrm{~g} /$ $\mathrm{L}$ or greater between 24 and $48 \mathrm{~h}$ of age [36]. In the current study, although serum IgG at $48 \mathrm{~h}$ after birth in TRT6 (14.8 g/L) or TRT12 (15.3 g/L) calves was less than that in TRT0 $(19.0 \mathrm{~g} / \mathrm{L})$, all of the calves had a 
serum IgG greater than $10 \mathrm{~g} / \mathrm{L}$ and achieved adequate passive transfer following the administration of colostrum [16]. It was likely that all the calves had the capability to prevent challenges from pathogens through adequate passive transfer. Therefore, the higher blood IgG concentration - well above the threshold of successful passive transfer (i.e., $10 \mathrm{~g} / \mathrm{L}$ ) - observed in TRT0 calves may not result in effects for the colonic mucosal immune system development in calves at 51 -h of age. Previous studies reported that the serum IgG concentration in dairy calves reached a maximum at $24 \mathrm{~h}$ of age and declined thereafter [37-39]. Since calves rely on colostral IgG for protection from pathogens until 3 or 4 weeks of age, when their own active immune system is functional [38], there would be a period before week 3 or 4 of age where blood IgG would be under the threshold of $10 \mathrm{~g} / \mathrm{L}$. Therefore, we speculate that the TRT0 calves may have greater IgG levels than TRT6 and TRT12 late in life when IgG concentrations decrease, such as later in week 3 or week 4 . Ultimately, this needs further investigation.

In addition, it is worth noting that the colon transcriptome varies among calves even when under the same dietary treatment and within similar dam parity. While there was no overall change in the expression level of the selected genes, some individuals exhibited substantial changes $(\triangle C P M$, a difference with the overall average), with both positive and negative responses $(\triangle \mathrm{CPM})$ occurring depending on selected genes (Additional file 7). Such large individual variation may lead to the lack of difference in the expression of immune related genes in the colon among different colostrum feeding strategies. Thus, there is a need to increase the amount of animals required in order to minimize the effects occurring from the animal itself and understand the actual impact of colostrum feeding time on the transcripotome profiles. In fact, variations among individual animals fed the same diet are commonly reported $[40,41]$. When feeding the same diet, the reasons behind animal variation are likely related to animal behavior, physiology, genetics, dam condition, and microbial community [40-43]. In the current study, when considering the parity of the dam on calf transcriptome profile, expression of genes RGS2 and CCL14 were different between calves from primiparous and multiparous cows within TRT0 or TRT6. The mechanisms behind how the expression of RGS2 or CCL14 were altered by parity are unclear. The inconsistency across treatments for changes in gene expression due to parity (i.e., altered RGS2 within TRT0, altered CCL14 within TRT6, and no change within TRT12) indicates that the comprehensive genetic background of the dam, including the parity, may come into play.

Increasing evidence has shown that early colonized microbiota in the gastrointestinal tract play a crucial role in the development of the mucosal immune system and influence newborn health $[44,45]$. In this study, a network-based analysis WGCNA, which combined the microbial abundance and transcriptome data in the colon with the same animal, confirmed that the immune related genes in the colon were associated with bacterial colonization, in particular with the abundance of Lactobacillus and $F$. prausnitzii, which were consistent with previous studies [32, 46]. In these studies, negative correlations between expression of mucosal TLRs and mucosa-attached bacteria were observed in young dairy calves. Moreover, it has been reported that $F$. prausnitzii displayed a negative association with calf diarrhea incidences [47] and the administration of Lactobacillus to newborn calves during the first week of life decreased diarrhea incidences [48]. Such findings suggest that a high abundance of $F$. prausnitzii or Lactobacillus during early life may play a vital role in maintaining colonic homeostasis and decreasing susceptibility to enteric infections in neonatal calves.

\section{Conclusion}

Our results showed that there were few changes on transcriptome profiles of the colon in 51-h age of calves fed the first colostrum meal at different time within $12 \mathrm{~h}$ after birth, suggesting that transcriptome profiles of the colon in neonatal calves are independent of the timing of the initial colostrum meal within $12 \mathrm{~h}$ after birth. However, it is noted that there was a large individual variation in the transcriptomic profiles among the neonatal calves. The potential for improving calf mucosal immune system development through feeding colostrum meals immediately after birth still needs to be defined using a larger number of animals or a prolonged sampling time. Regardless, the transcriptome analysis provides a molecular understanding of the colonic biological function in neonatal calves and gene co-expression analysis extends knowledge on how gut tissue can interact with its luminal bacterial community, which may help to develop future nutritional management strategies for calf performance.

\section{Additional files}

Additional file 1: List of immune related genes in the colon of 51-h age of dairy calves and the gene list obtained from ImmPort. (XLSX 12419 kb)

Additional file 2: Sample information details and sequencing results mapped to reference genome by Tophat2. (XLSX $10 \mathrm{~kb})$

Additional file 3: List of core genes in the colon. (XLSX $785 \mathrm{~kb}$ ) Additional file 4: Clustering dendrogram of genes showing module membership in colours. (TIFF 946 kb)

Additional file 5: WGCNA identification of colonic gene modules correlated with the bacterial abundance. The module-trait relationships indicates the correlation coefficients and $p$-values (in the brackets). The color scale bar shown in the right represents the Pearson correlation ranging from -1 (green) to 1 (red). The bacterial population examined 
were Lactobacillus from the colon content (lac_C_P), Lactobacillus from the attached colon (lac_T_P), Faecali bacterium prausnitzii from the colon content (fea_C_P), Faecali bacterium prausnitzii from the attached colon (fea_T_P), Bifidobacterium from the colon content (bif_C_P), Bifidobacterium from the attached colon (bif $T$ P P), which were shown on the bottom. (PDF $8 \mathrm{~kb}$ )

Additional file 6: List of genes in significant modules identified using WGCNA. (XLSX $108 \mathrm{~kb}$ )

Additional file 7: Animal variations of 12 representative immune genes. (PDF $354 \mathrm{~kb}$ )

\section{Abbreviations}

A2M: Alpha-2-macroglobulin; ACTG: Actin gamma; AHNAK: AHNAK nucleoprotein; BCR: Breakpoint cluster region; C: Multiparous cows; CALR: Calreticulin; CANX: Calnexin; CCL: Chemokine (C-C motif) ligand; CLDN: Claudin; CPM: Counts per million; DE gene: Differentially expressed gene; DES: Desmin; FC: Fold change; FDR: False discovery rate; GO: Gene ontology; H: Primiparous heifers; HSPA: Heat shock protein family A; Ig: Immunoglobulins; IL: Interleukin; LRP1: LDL receptor related protein; NFKB: Nuclear factor kappa B; PANTHER: Protein annotation through evolutionary relationship; PCA: Principal component analysis;

PGLYRP: Peptidoglycan recognition protein; RGS: Regulator of G protein signaling; RNA-seq: RNA-sequencing; SCFA: Short chain fatty acid; SLC40A1: Solute carrier family 40 member 1; STAT: Signal transducers and activators of transcription; TCR: T cell receptor; TGF: Transforming growth factor; TLR: Toll-like receptors; TNF: Tumor necrosis factor; TPM: Tropomyosin; TRT0: Fed immediately after birth; TRT12: Fed at $12 \mathrm{~h}$ after birth; TRT6: Fed at $6 \mathrm{~h}$ after birth; WGCNA: Weighted gene co-expression network analysis

\section{Acknowledgements}

The authors thank the staff of Dairy Research and Technology Centre (University of Alberta) for their assistance in keeping animals. We thank the lab members of Dr. Steele and Dr. Guan for animal trial and sampling and Ms. Y. Chen for advice on RNA sequencing libraries construction. Special thanks go to the Pioneer Hundred Talents Program of the Chinese Academy of Science for supporting the research career of Dr. Z.X. He.

\section{Funding}

This project is supported by Alberta Livestock and Meat Agency Ltd. (2015B013), Alberta Milk, The Saskatoon Colostrum Co. Ltd. and the Natural Sciences and Engineering Research Council of Canada.

\section{Availability of data and materials}

All the RNA-Seq data were deposited in the publicly available NCBI's Gene Expression Omnibus Database (https://www.ncbi.nlm.nih.gov/geo/). The data are accessible through GEO Series accession number GSE113264 (https:// www.ncbi.n/m.nih.gov/geo/query/acc.cgi?acc=GSE113264). The datasets supporting the conclusions of this article are included within the article and its additional files.

\section{Authors' contributions}

MS, LG, AF and YS designed the experiment; ZH, AF and YS conducted the animal trial, collected samples, sequencing data analysis pipeline and data interpretation; ZH prepared libraries, analyzed data and draft the writing; MS and LG revised the manuscript. All authors contributed to manuscript preparation and writing. All authors read and approved the final manuscript.

\section{Ethics approval and consent to participate}

The Livestock Care Committee of the University of Alberta reviewed and approved all procedures for the animal trial (AUP00001595).

\section{Consent for publication}

Not applicable.

\section{Competing interests}

The authors declare that they have no competing interests.

\section{Publisher's Note}

Springer Nature remains neutral with regard to jurisdictional claims in published maps and institutional affiliations.

\section{Author details}

${ }^{1}$ Key Laboratory of Agro-ecological Processes in Subtropical Region, Institute of Subtropical Agriculture, Chinese Academy of Sciences, Changsha, Hunan 410125, People's Republic of China. ${ }^{2}$ Department of Agriculture, Food and Nutritional Sciences, University of Alberta, Edmonton T6G 2P5, Canada.

Received: 21 April 2018 Accepted: 14 August 2018

Published online: 28 August 2018

\section{References}

1. Borghesi J, Mario LC, Nogueira MR, Favaron PO, Miglino MA. Immunoglobulin transport during gestation in domestic animals and humans - a review. Open J Anim Sci. 2014;04:323-36.

2. Davidson JN, Yancey SP, Campbell SG, Warner RG. Relationship between serum immunoglobulin values and incidence of respiratory disease in calves. J Am Vet Med Assoc. 1981;179:708-10.

3. Robison JD, Stott GH, Denise SK. Effects of passive immunity on growth and survival in the dairy heifer. J Dairy Sci. 1988;71:1283-7.

4. Tyler JW, Hancock DD, Thorne JG, Gay CC, Gay JM. Partitioning the mortality risk associated with inadequate passive transfer of colostral immunoglobulins in dairy calves. J Vet Intern Med. 1999;13:335-7.

5. Lora I, Gottardo F, Contiero B, Ava BD, Bonfanti L, Stefani A, Barberio A. Association between passive immunity and health status of dairy calves under 30 days of age. Prev Vet Med. 2018;152:12-5.

6. Godden S. Colostrum management for dairy calves. Vet Clin North Am Food Anim Pract. 2008;24:19-39.

7. Olson DP, Papasian CJ, Ritter RC. The effects of cold stress on neonatal calves. II. Absorption of colostral immunoglobulins. Can J Comp Med. 1980; 44:19-23.

8. Chigerwe M, Tyler JW, Schultz LG, Middleton JR, Steevens BJ, Spain JN. Effect of colostrum administration by use of oroesophageal intubation on serum IgG concentrations in Holstein bull calves. Am J Vet Res. 2008;69: $1158-63$.

9. Blum JW, Hammon $\mathrm{H}$. Colostrum effects on the gastrointestinal tract, and on nutritional, endocrine and metabolic parameters in neonatal calves. Livest Prod Sci. 2000;66:151-9.

10. Baumrucker $C R$, Blum JW. Effects of dietary recombinant human insulin-like growth factor-I on concentrations of hormones and growth factors in the blood of newborn calves. J Endocrinol. 1994;140:15-21.

11. Koldovský O. Search for role of milk-borne biologically active peptides for the suckling. J Nutr. 1989;119:1543-51.

12. Wang HB, Wang PY, Wang $X$, Wan YL, Liu YC. Butyrate enhances intestinal epithelial barrier function via up-regulation of tight junction protein Claudin-1 transcription. Dig Dis Sci. 2012;57:3126-35.

13. Arpaia N, Campbell C, Fan X, Dikiy S, van der Veeken J, deRoos P, Liu H, Cross JR, Pfeffer K, Coffer PJ, Rudensky AY. Metabolites produced by commensal bacteria promote peripheral regulatory T-cell generation. Nature. 2013;504:451-5.

14. Song Y, Malmuthuge N, Steele MA, Guan LL. Shift of hindgut microbiota and microbial short chain fatty acids profiles in dairy calves from birth to preweaning. FEMS Microbiol Ecol. 2018;94 https://doi.org/10.1093/femsec/fix179.

15. Canadian Council on Animal Care (CCAC). In: Olfert ED, Cross BM, McWilliams AA, editors. Guide to the care and use of experimental animals, vol. 1. 2nd ed. Ottawa: CCAC; 1993.

16. Fischer AJ, Song Y, He Z, Haines DM, Guan LL, Steele MA. Effect of delaying colostrum feeding on passive transfer and intestinal bacterial colonization in neonatal male Holstein calves. J Dairy Sci. 2018;101:3099-109.

17. Sequencing BG, Consortium A, Elsik CG, Tellam RL, Worley KC, Gibbs RA, Muzny DM, Weinstock GM, Adelson DL, Eichler EE. The genome sequence of taurine cattle: a window to ruminant biology and evolution. Science. 2009;324:522.

18. Kim D, Pertea G, Trapnell C, Pimentel H, Kelley R, Salzberg SL. TopHat2: accurate alignment of transcriptomes in the presence of insertions, deletions and gene fusions. Genome Biol. 2013;14:R36.

19. Li H, Handsaker B, Wysoker A, Fennell T, Ruan J, Homer N, et al. The sequence alignment/map format and SAMtools. Bioinformatics. 2009;25: 2078-9. 
20. Anders S, Pyl PT, Huber W. HTSeq--a Python framework to work with highthroughput sequencing data. Bioinformatics. 2015;31:166-9.

21. Mi H, Thomas P. PANTHER pathway: an ontology-based pathway database coupled with data analysis tools. Methods Mol Biol. 2009;563:123-40.

22. Robinson MD, Mccarthy DJ, Smyth GK. edgeR: a Bioconductor package for differential expression analysis of digital gene expression data. Bioinformatics. 2010;26:139-40.

23. Bhattacharya S, Andorf S, Gomes L, Dunn P, Schaefer H, Pontius J, Berger P, Desborough V, Smith T, Campbell J. ImmPort: disseminating data to the public for the future of immunology. Immunol Res. 2014;58:234-9.

24. Eicher SD, Mcmunn KA, Hammon HM, Donkin SS. Toll-like receptors 2 and 4, and acute phase cytokine gene expression in dexamethasone and growth hormone treated dairy calves. Vet Immunol Immunopathol. 2004;98:115.

25. Axel H, Allison MC, Neil W, Susanne M, Jane K, Caroline W, Crookenden MA, Mitchell MD, Stuart M, Kate W. Grazing dairy cows had decreased interferon- $\gamma$, tumor necrosis factor, and interleukin-17, and increased expression of interleukin-10 during the first week after calving. J Dairy Sci. 2015;98:937-46.

26. Malmuthuge N, Li M, Goonewardene LA, Oba M, Guan LL. Effect of calf starter feeding on gut microbial diversity and expression of genes involved in host immune responses and tight junctions in dairy calves during weaning transition. J Dairy Sci. 2013;96:3189-200.

27. Osorio JS, Trevisi E, Ballou MA, Bertoni G, Drackley JK, Loor JJ. Effect of the level of maternal energy intake prepartum on immunometabolic markers, polymorphonuclear leukocyte function, and neutrophil gene network expression in neonatal Holstein heifer calves. J Dairy Sci. 2013;96:3573-87.

28. Langfelder P, Horvath S. WGCNA: an R package for weighted correlation network analysis. BMC Bioinf. 2008;9:559.

29. Byrne CS, Chambers ES, Morrison DJ, Frost G. The role of short chain fatty acids in appetite regulation and energy homeostasis. Int J Obes. 2015;39: 1331

30. Schumann A, Nutten S, Donnicola D, Comelli EM, Mansourian R, Cherbut C, Corthesytheulaz I, Garciarodenas C. Neonatal antibiotic treatment alters gastrointestinal tract developmental gene expression and intestinal barrier transcriptome. Physiol Genomics. 2005;23:235-45.

31. Mcghee JR, Kohtaro F. Inside the mucosal immune system. PLoS Biol. 2012; 10:e1001397.

32. Liang G, Malmuthuge N, Bao H, Stothard P, Griebel PJ, Guan LL. Transcriptome analysis reveals regional and temporal differences in mucosal immune system development in the small intestine of neonatal calves. BMC Genomics. 2016:17:602.

33. Cho Y, Yoon KJ. An overview of calf diarrhea - infectious etiology, diagnosis, and intervention. J Vet Sci. 2014;15:1.

34. Tizard. Immunity in the fetus and newborn. In: Veterinary immunology, an introduction. 4th ed. Philadelphia: WB. Sanders Company; 1992. p. 248-60.

35. Tourneur E, Chassin C. Neonatal immune adaptation of the gut and its role during infections. Clin Dev Immunol. 2013;2013:270301.

36. Weaver DM, Tyler JW, Vanmetre DC, Hostetler DE, Barrington GM. Passive transfer of colostral immunoglobulins in calves. J Vet Intern Med. 2000;14: 569-77

37. Burton JL, Kennedy BW, Burnside EB, Wilkie BN, Burton JH. Variation in serum concentrations of immunoglobulins $\mathrm{G}, \mathrm{a}$, and $\mathrm{M}$ in Canadian Holstein-Friesian calves. J Dairy Sci. 1989;72:135-49.

38. Conneely M, Berry DP, Murphy JP, Lorenz I, Doherty ML, Kennedy E. Effect of feeding colostrum at different volumes and subsequent number of transition milk feeds on the serum immunoglobulin $\mathrm{G}$ concentration and health status of dairy calves. J Dairy Sci. 2014:97:6991-7000.

39. Morin DE, Mccoy GC, Hurley WL. Effects of quality, quantity, and timing of colostrum feeding and addition of a dried colostrum supplement on immunoglobulin G1 absorption in Holstein bull calves. J Dairy Sci. 1997;80: 747-53.

40. Gao X, Oba M. Relationship of severity of subacute ruminal acidosis to rumen fermentation, chewing activities, sorting behavior, and milk production in lactating dairy cows fed a high-grain diet. J Dairy Sci. 2014;97: 3006-16.

41. Rius A, Kittelmann S, Macdonald K, Waghorn G, Janssen P, Sikkema E. Nitrogen metabolism and rumen microbial enumeration in lactating cows with divergent residual feed intake fed high-digestibility pasture. J Dairy Sci. 2012;95:5024-34

42. Hernandez-Sanabria E, Goonewardene LA, Wang Z, Durunna ON, Moore SS. Impact of feed efficiency and diet on adaptive variations in the bacterial community in the rumen fluid of cattle. Appl Environ Microbiol. 2012;78: 1203-14.

43. Kong RS, Liang G, Chen $Y$, Stothard P. Transcriptome profiling of the rumen epithelium of beef cattle differing in residual feed intake. BMC Genomics. 2016;17:592.

44. Fouhy F, Guinane CM, Hussey S, Wall R, Ryan CA, Dempsey EM, Murphy B, Ross RP, Fitzgerald GF, Stanton C. High-throughput sequencing reveals the incomplete, short-term recovery of infant gut microbiota following parenteral antibiotic treatment with ampicillin and gentamicin. Antimicrob Agents Chemother. 2012;56:5811-20.

45. Malmuthuge N, Griebel PJ. The gut microbiome and its potential role in the development and function of newborn calf gastrointestinal tract. Front Vet Sci. 2015;2:36

46. Malmuthuge N, Li M, Fries P, Griebel PJ. Regional and age dependent changes in gene expression of toll-like receptors and key antimicrobial defence molecules throughout the gastrointestinal tract of dairy calves. Vet Immunol Immunopathol. 2012:146:18-26.

47. Oikonomou G, Teixeira AGV, Foditsch C, Bicalho ML, Machado VS, Bicalho RC. Fecal microbial diversity in pre-weaned dairy calves as described by pyrosequencing of metagenomic 165 rDNA. Associations of Faecalibacterium species with health and growth. PLoS One. 2013;8:e63157.

48. Abe F, Ishibashi N, Shimamura S. Effect of administration of bifidobacteria and lactic acid bacteria to newborn calves and piglets. J Dairy Sci. 1995;78: 2838-46.
Ready to submit your research? Choose BMC and benefit from:

- fast, convenient online submission

- thorough peer review by experienced researchers in your field

- rapid publication on acceptance

- support for research data, including large and complex data types

- gold Open Access which fosters wider collaboration and increased citations

- maximum visibility for your research: over $100 \mathrm{M}$ website views per year

At BMC, research is always in progress.

Learn more biomedcentral.com/submissions 